\title{
Effect of CVD graphene on hydrate formation of natural gas
}

\author{
Mohsen Hosseini • Ahmad Ghozatloo • \\ Mojtaba Shariaty-Niassar
}

Received: 20 December 2014 / Accepted: 16 February 2015/Published online: 4 March 2015

(C) The Author(s) 2015. This article is published with open access at Springerlink.com

\begin{abstract}
In this study, graphene nano-sheets were grown and synthesized on copper foils and the structure was analyzed with XRD, FTIR and SEM. After that, $1 \mathrm{wt} \%$ low-layer graphene nano-sheets were added to water and stabilized by sodium dodecyl sulfate (SDS). Prepared nano-fluid was then used in hydrate formation of natural gas at $1000 \mathrm{psig}$ and $4{ }^{\circ} \mathrm{C}$. The results were compared with a SDS/water system and observed that graphene nanosheets had reduced the dissolution time $19.2 \%$, induction time $54.7 \%$ and the amount of gas storage is increased $7.6 \%$. Hydrate stability test showed that the hydrate stability is also increased $5.9 \%$.
\end{abstract}

Keywords Hydrate + Natural gas - Graphene nanosheets $\cdot$ Storage $\cdot$ Induction time

\section{Introduction}

Hydrate is an ice-like solid which can be made if water could be in contact with high pressure and low temperature gases. The demand for gas distribution to different regions and also long distances between them made scientists to develop new solutions for gas distribution; among them we can point to LNG, PLNG, GTL, ANG, GTW, CNG and

M. Hosseini · A. Ghozatloo · M. Shariaty-Niassar Transport Phenomena and Nanotechnology Laboratory, Department of Chemical Engineering, School of Engineering, University of Tehran, P.O. Box: 11155-4563, Tehran, Iran

A. Ghozatloo $(\square)$

Nano Technology Research Center, Research Institute of Petroleum Industry (RIPI), West Blvd. Azadi Sport Complex, P.O. Box: 14665-137, Tehran, Iran

e-mail: ghozatlooa@ripi.ir also NGH (natural gas hydrates). NGH is new method in natural gas storage and distribution [1]. Many researches were made on NGH in recent years [2-6]. Low speed of hydrate formation process and lack of hydrate stability are of the major problems against the industrial NGH cycle. Scientists have used different promoters to improve the natural gas hydrate formation process (HFP) which most of them were hydrophilic surfactants [7]. These promoters could increase hydrate formation speed and gas storage capacity [8-17].

There are four factors together needed in HFP: low temperature, high pressure, presence of water and presence of gas. Effective parameters in HFP could be divided to two parts of process parameters and the parameters related to the promoter. Major process parameters are temperature, pressure, speed of the mixer, amount of fluid used, and in some cases volume of the reactor [18]. Major nano-fluid promoter-related parameters could be heat transfer characteristics, geometry, size, porosity, concentration and stability of nano-particles.

As the HFP is an interfacial phenomenon, any operant that could increase heat transfer and mass transfer in the interfacial of fluid/gas definitely can increase speed of HFP and also the gas stored in hydrates [18]. Therefore, the issues can be solved by enhancing heat transfer and mass transfer. Using a mixer may be suitable for enhancing mass transfer, as it brings turbulency into the system. Fltp flcp mixing [19] and magnetic stirring [20] were also used in researches for enhancing mass transfer. On the other hand, nano-particles can prepare more active sites for nucleation of gas because of their nonmetric dimensions. HFP is an exothermic process and heat transfer plays an important role in it. Hydrates are stable in high pressure and low temperatures; so anything that can crash this thermodynamic state will make hydrates unstable; therefore it is needed to somehow send out the heat 
generated by HFP so that the process can continue with enough speed. A jacketed reactor can be suitable for sending the heat out of the system but to enhance the heat transfer inside the reactor, it is suggested to use nano-particles as they turn water into a nano-fluid with a high heat transfer coefficient [21]. Using a nano-fluid instead of water in the reactor may be effective in HFP speed. In this study, CVD graphene nano-sheets were used in natural gas HFP. The idea is made from their high heat transfer coefficient which is around $5000 \mathrm{~W} / \mathrm{m} \mathrm{K}$ [21]. Therefore, it is suggested to prepare a stable nano-fluid using SDS as stabilizer, and use the nano-fluid instead of water for facilitating the HFP. Surfactants do have an effect on the methane mole fraction in the bulk liquid during hydrate growth [6]. They could achieve an increase in hydrate former mole fraction by reducing the interphase mass transfer resistance at the gas/ liquid interface. Surfactants can reduce surface tension under hydrate forming conditions [22]. Decreasing the adhesion forces between hydrate particles or hydrophobic microdomains at the hydrate/liquid interface are other possible mechanisms [23]. It has also been observed that using surfactant leads to looser hydrogen bonding that is an indicator for facilitating hydrate growth [24]. Furthermore, Banks showed that the underlying surface will electrochemically catalyze the response [25-27], therefore, it can be concluded that adding SDS for stabilizing the graphene nano-fluid may bring an electro-catalytic effect on HFP process.

\section{Synthesis of graphene nano-sheets and used materials}

Graphene nano-sheets were grown and synthesized on copper foils by CVD method. First, $25 \mu \mathrm{m}$ copper foils were loaded into a quartz tube reactor, and then the reactor was put into a furnace. Pressure inside the reactor was decreased to 1-10 m Torr by a vacuum pump and then $2 \mathrm{~cm}^{3} /$ min helium flows into the reactor. Furnace was heated up to $1050{ }^{\circ} \mathrm{C}$ and was kept in this temperature for $40 \mathrm{~min}$. After that, $35 \mathrm{~cm}^{3} /$ min methane was added to hydrogen and their mixture flowed into the reactor for $15 \mathrm{~min}$. A quick cooling method was used $180{ }^{\circ} \mathrm{C} / \mathrm{min}$ after the growth and the helium flow was continued throughout the cooling process. CVD grown graphene nano-sheets could be easily separated from the copper foil $[6,21,22]$. SDS with $99 \%$ purity was bought from Merck Co. Germany. Deionized water was bought from Zolal-Bahr Co. and natural gas was supplied from Air Product Co. and was used as reactor feed.

\section{Graphene nano-sheets structure analysis}

SEM, FTIR and XRD analyzes were carried out to study the structure of synthesized CVD graphene nano-sheets.
The crystallinity of the samples was studied using XRD. Figure 1 shows XRD spectrum of CVD-synthesized graphene nano-sheets.

Graphene XRD spectrum indicating peak is a short and relatively wide peak at $2 \theta=26.5^{\circ}$ which corresponds to the (002) reflection of graphite, with spacing of $0.34 \mathrm{~nm}$ [28] that can be well observed in Fig. 1. This result is consistent with the other researches [29, 30]. The peak of CVD graphene nano-sheets at $26.5^{\circ}$ is slightly wide which is because of its small flake sizes [31]. Graphene nanosheets were completely crushed and mixed with potassium bromide powder and the mixture was then pressed into tablets and put into FTIR spectrometry device. The functionalization and chemical structure of CVD graphene was analyzed by FTIR. Typically 100 scans over the range $4000-500 \mathrm{~cm}^{-1}$ were taken with a resolution of $2 \mathrm{~cm}^{-1}$ [21]. Figure 2 shows the FTIR spectrum of CVD-synthesized graphene nano-sheets.

FTIR indicating peaks of CVD graphene are clearly evident at 1574 and $3070 \mathrm{~cm}^{-1}$ corresponding to stretching of $\mathrm{C}=\mathrm{C}$ bonds and $\mathrm{C}-\mathrm{H}$ stretching vibration of aromatic compound, respectively [30, 32]. Existence of $\mathrm{C}=\mathrm{C}$ bonds confirms the aromatic structure of CVD graphene nanosheets. Figure 2 shows that the synthesized graphene is pure due to absence of oxygen-containing functional groups. The result taken from FTIR is consistent with XRD result, confirming the synthesis of graphene nano-sheets. Raman spectroscopy was employed to evaluate the quality and layer number of the graphene. The D band corresponds to the defects induced in the graphitic structure and functional groups attached to the surface of graphene sheets, while the $\mathrm{G}$ band corresponds to the in-plane vibrations of the graphitic structure [33].

The typical features for carbon in Raman spectra are the $\mathrm{G}$ band around $1582 \mathrm{~cm}^{-1}$ and the $\mathrm{D}$ band around $1350 \mathrm{~cm}^{-1}$ [28]. The $2 \mathrm{D}$ band is a key mode in identification of number of graphene layers. According to its position, shape and intensity, a thickness of graphene may be

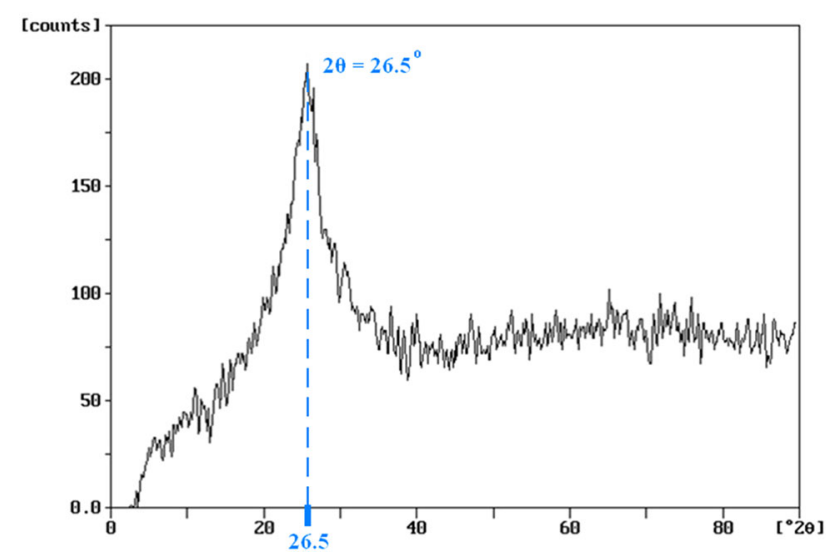

Fig. 1 XRD spectrum of synthesized CVD graphene nano-sheets 
Fig. 2 FTIR spectrum of CVDsynthesized graphene nanosheets

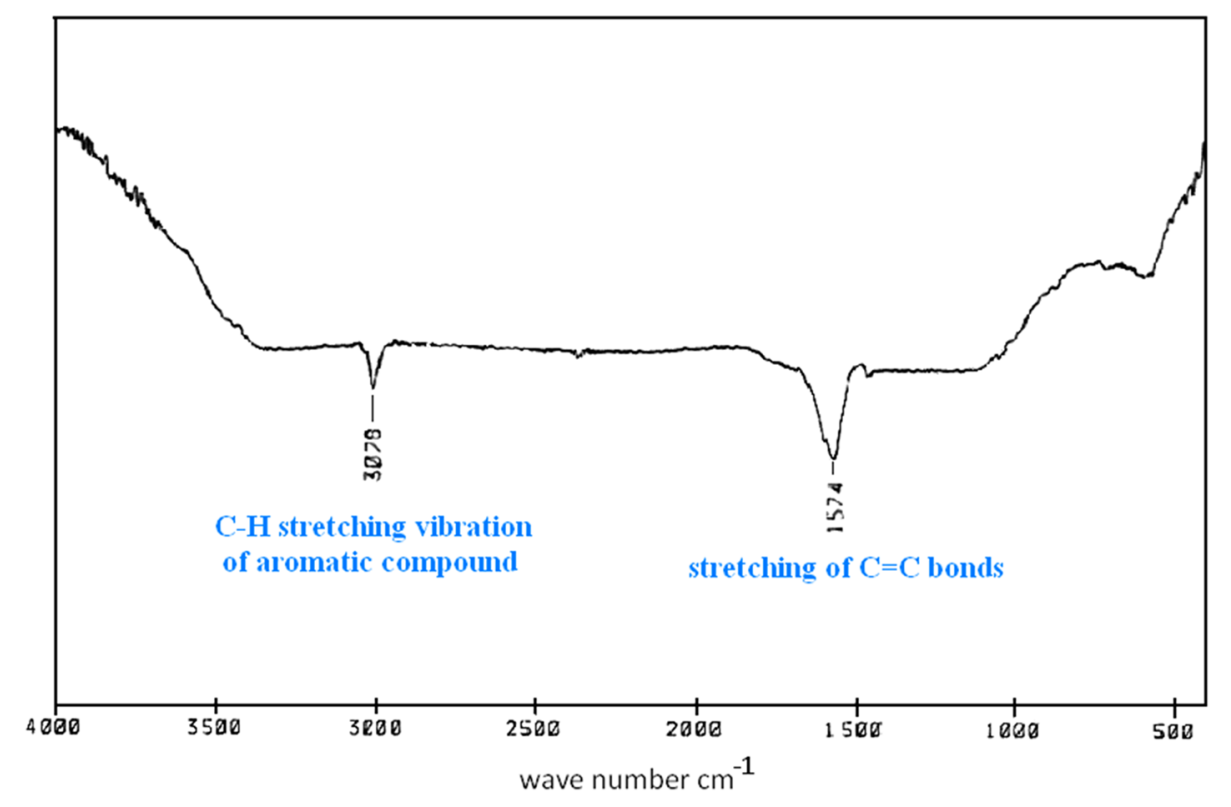

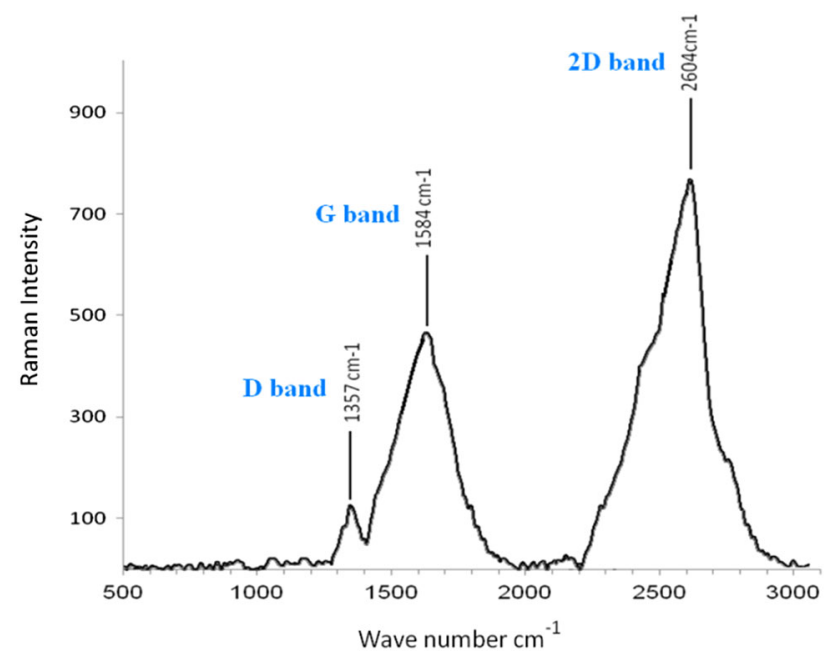

Fig. 3 Raman spectrum of CVD-synthesized graphene nano-sheets

determined. 2D band of single-layer graphene is composed of a single 2D band, positioned at roughly $2600 \mathrm{~cm}^{-1}$ [34]. Figure 3 shows Raman spectra of CVD graphene nanosheets. The Raman spectrum of the CVD graphene displays a strong $\mathrm{G}$ band at $1584 \mathrm{~cm}^{-1}$, a weak $\mathrm{D}$ band at $1357 \mathrm{~cm}^{-1}$. It is also seen that single $2 \mathrm{D}$ band appears at $2602 \mathrm{~cm}^{-1}$ attributed to single-layer graphene. It is known that a ratio between the intensities of the $D$ and the $G$ bands $\left(I_{\mathrm{D}} / I_{\mathrm{G}}\right)$ determines relative defect content in the carbon lattice [34]. The Raman spectra show a week D band that confirms the small amount of defects.

Transmission electron microscopy is also a powerful technique used extensively to provide definitive identification of graphene materials. Figure 4 shows the TEM

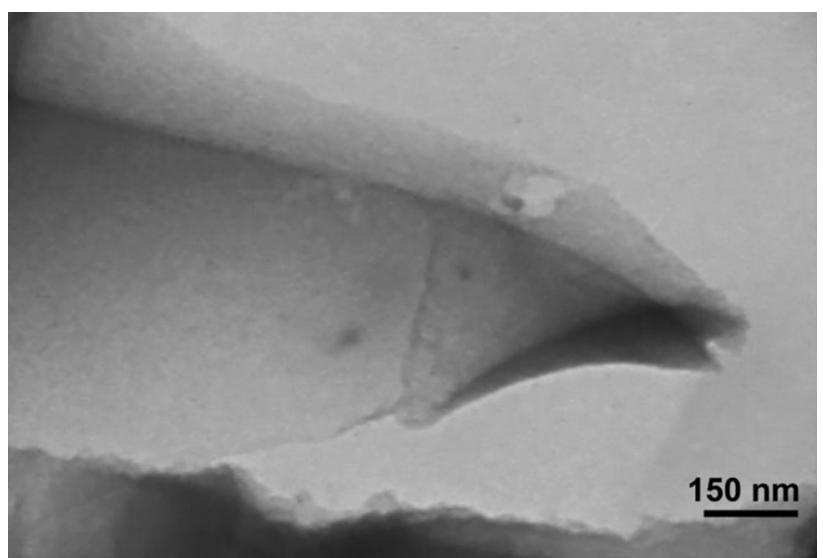

Fig. 4 TEM images of CVD-synthesized graphene nano-sheets

image of the synthesized graphene. TEM image indicates the sheet morphology of synthesized graphene. The most transparent regions are likely to be monolayer graphene nano-sheets which is in agreement with the results taken from the Raman spectroscopy. TEM observation also reveals that the synthesized graphene is pure due to the uniformity of the graphene nano-sheets. Closer observation of the TEM shows the folding at the edges of graphene nano-sheets.

\section{Preparing nano-fluid and hydrate formation apparatus}

To prepare a $1 \mathrm{wt} \%$ nano-fluid, $1 \mathrm{~g}$ of graphene nanosheets were added to $99 \mathrm{~g}$ deionized water. $1.5 \mathrm{~g}$ SDS was added slowly to the fluid while mixing it to obtain the stability of graphene nano-sheets in water. This is one of 
the general methods to avoid sedimentation of nano-particles. Addition of surfactant can improve the stability of nano-particles in aqueous suspensions. The reason is that the hydrophobic surfaces of nano-sheets are modified to become hydrophilic and vice versa for non-aqueous liquids. A repulsion force between suspended particles is caused by the zeta potential which will rise due to the surface charge of the particles suspended in the base fluid $[35,36]$. The system was then put in ultrasonic bath for $45 \mathrm{~min}$. The stability of the nano-fluid was studied after $48 \mathrm{~h}$ and after ensuring it, nano-fluid was used in HFP.

HFP was performed in a $600 \mathrm{ml}$ steel Parr reactor. To control temperature of the system, the reactor was placed in a Teflon jacket and water/ethylene-glycol solution was used as coolant in the jacket which was circulated by a Lauda RE10 circulator with an accuracy of $\pm 0.01 \mathrm{~K}$. Two needle valves are placed on the door of reactor for entry and exit of the gas and the reactor is equipped with a mechanical four blade mixer which is controlled in range of 100-1000 rpm. Schematic of HFP apparatus is shown in Fig. 5.

Reactor temperature is measured with a temperature sensor with an accuracy of $0.01 \mathrm{~K}$, equipped with temperature electrical pulse saving system and the data will be sent to the computer. Reactor pressure is measured with a BD SENSORS 28/600 pressure sensor in range of 0-140 bar with an accuracy of 0.001 bar, equipped with pressure electrical pulse saving system and sending the measurement instantaneously the computer.

Initially the reactor was washed, cleaned and dried completely. The reactor was then charged with $100 \mathrm{~g}$ of CVD graphene/water nano-fluid. Then, a sufficient amount of natural gas was injected into the reactor so that the air trapped inside the reactor completely evacuated. After that, the relief valve was closed and natural gas was injected into it until the pressure of the reactor reached 1000 psig. Reactor temperature was slightly reduced by setting the temperature of cooling system at $4{ }^{\circ} \mathrm{C}$.

Mole number of natural gas present in the rector at any time can be calculated by Eq. (1) where $\mathrm{z}$ is the compressibility factor, calculated by Peng-Robinson equation of state. Initial amount of natural gas present in the reactor was calculated to be $1.587 \mathrm{~mol}$. Over the time, pressure drop in the reactor is measured and recorded by the computer. Pressure drop observed in the reactor can be related to dissolution of natural gas in water and also formation and growth of natural gas hydrates. After some time, no

Fig. 5 Schematic of HFP apparatus

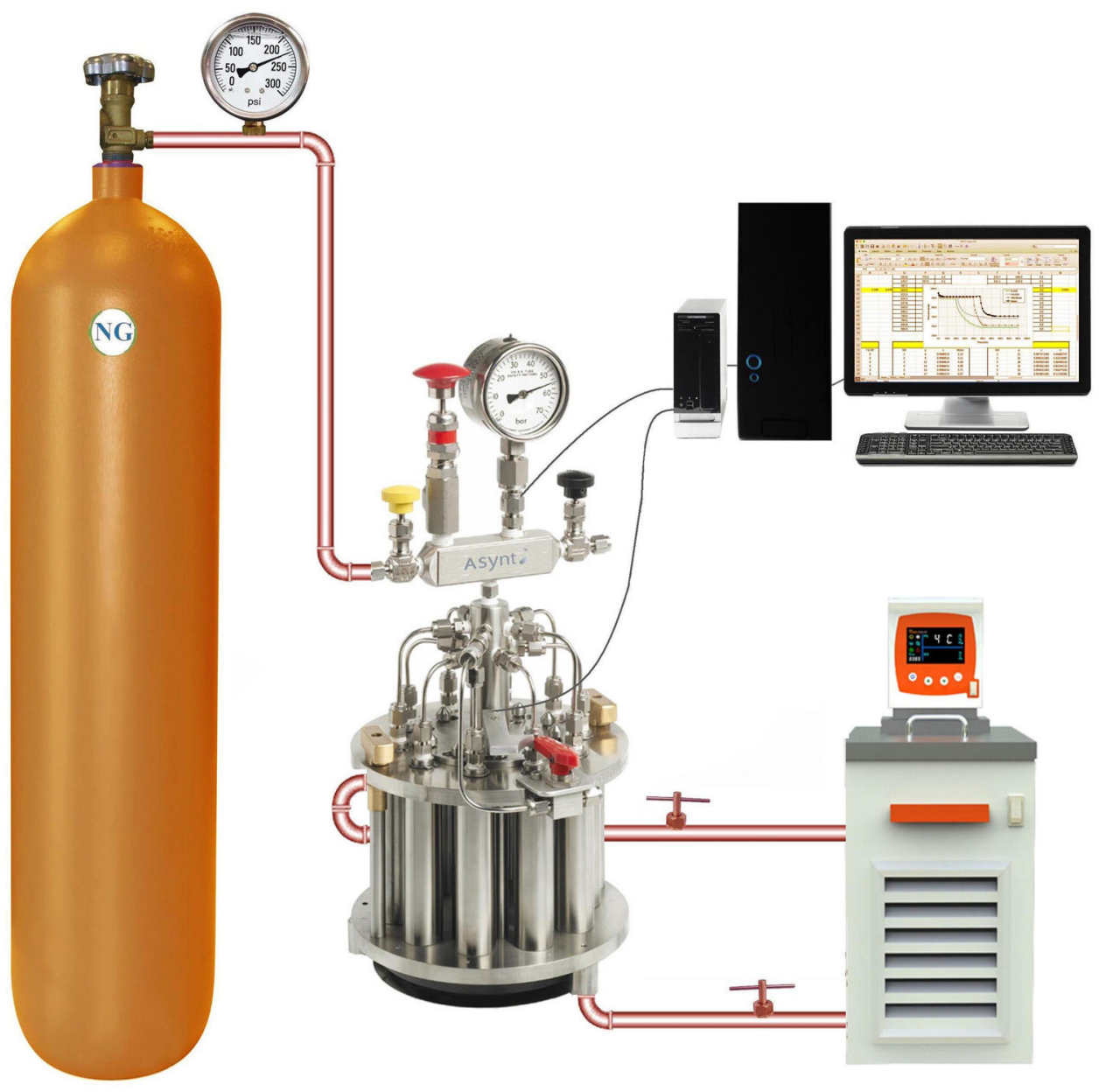


more pressure drop is observed in the reactor which shows that HFP is finished.

$n=P V / \mathrm{zRT}$

One of the major parameters in gas storage and distribution by hydrates is the storage capacity. This parameter is normally defined by the ratio of amount of gas stored in hydrates in standard condition, to the volume of the hydrates. Storage capacity at standard condition $(P=1 \mathrm{~atm}$ and $T=15^{\circ} \mathrm{C}$ ) can be calculated by Eq. (1). By substituting Eqs. (2) and (3) in Eqs. (1) and (4) shows that the storage capacity is related to $\rho$ (hydrate density), c (mole fraction of gas in hydrates) and MW (molecular weight of hydrates).

$n=m / \mathrm{MW}$

$\rho=m / V_{\mathrm{H}}$

$V / V_{\mathrm{H}}=23.643(\rho . c / \mathrm{MW})$

Methane hydrate is formed in structure I with a density of $913 \mathrm{~kg} / \mathrm{m}^{3}$ [37], molecular weight of $17.74 \mathrm{~kg} / \mathrm{kmol}$ [37] and mole fraction of methane in hydrates $14.1 \%$ [38]. In transportation and distribution of natural gas, it is better to storage pure methane in hydrates. By substituting the above data into the Eq. (4), maximum storage capacity in natural gas HFP will be calculated $V / V_{\mathrm{H}}=171.6$. The word "maximum" eventually refers to storing the pure methane.

Stability of the hydrates is another major parameter in gas storage and distribution by hydrates. To obtain stability, hydrate temperature should be reduced to $-10{ }^{\circ} \mathrm{C}$ [39]. Therefore, in HFP, after formation of hydrates, temperature is reduced to $-10{ }^{\circ} \mathrm{C}$ for $10 \mathrm{~h}$. Relief valve is opened then so that excess gas exits the reactor. When pressure of reactor reached $1 \mathrm{~atm}$, relief valve will be closed and pressure of the system is measured for $12 \mathrm{~h}$. Using Eq. (1) the amount of gas escaped from the hydrates can be calculated and reported as hydrate decomposition percentage.

\section{Results and discussion}

HFP in presence of graphene nano-sheets/water

Before using nano-fluid in HFP of natural gas, a blank solution of SDS/water was used in HFP under the same conditions. Graphene nano-fluid was then used in HFP of natural gas. Figure 6 shows the variation of temperature and pressure over the time in the reactor.

It is observed from Fig. 5 that the pressure was reduced to 931 psig initially. The pressure drop over the first 31 min may be caused by partial dissolution of natural gas in nano-fluid and also reduction of the temperature inside the reactor. The amount of gas remained in the reactor was calculated by Eq. (1) which is $1.547 \mathrm{~mol}$; therefore, $0.040 \mathrm{~mol}$ of natural gas has entered into the nano-fluid to participate in nucleation process. It is also observed in Fig. 6 that after $191 \mathrm{~min}$, pressure in the reactor was dropped; while temperature of the reactor was increased due to exothermic hydrate formation phenomenon. Therefore, the induction time for graphene nano-sheets/water can be calculated using Eq. (5) as follows:

$t_{\mathrm{i}_{\text {graphene nano-sheets/water }}}=191-31=160 \mathrm{~min}$

Pressure of the reactor was constant over 160 min while the temperature of the system was fixed at $4{ }^{\circ} \mathrm{C}$ due to the
Fig. 6 Variation of temperature and pressure over the time in the reactor for graphene nanosheets/water and SDS/water samples

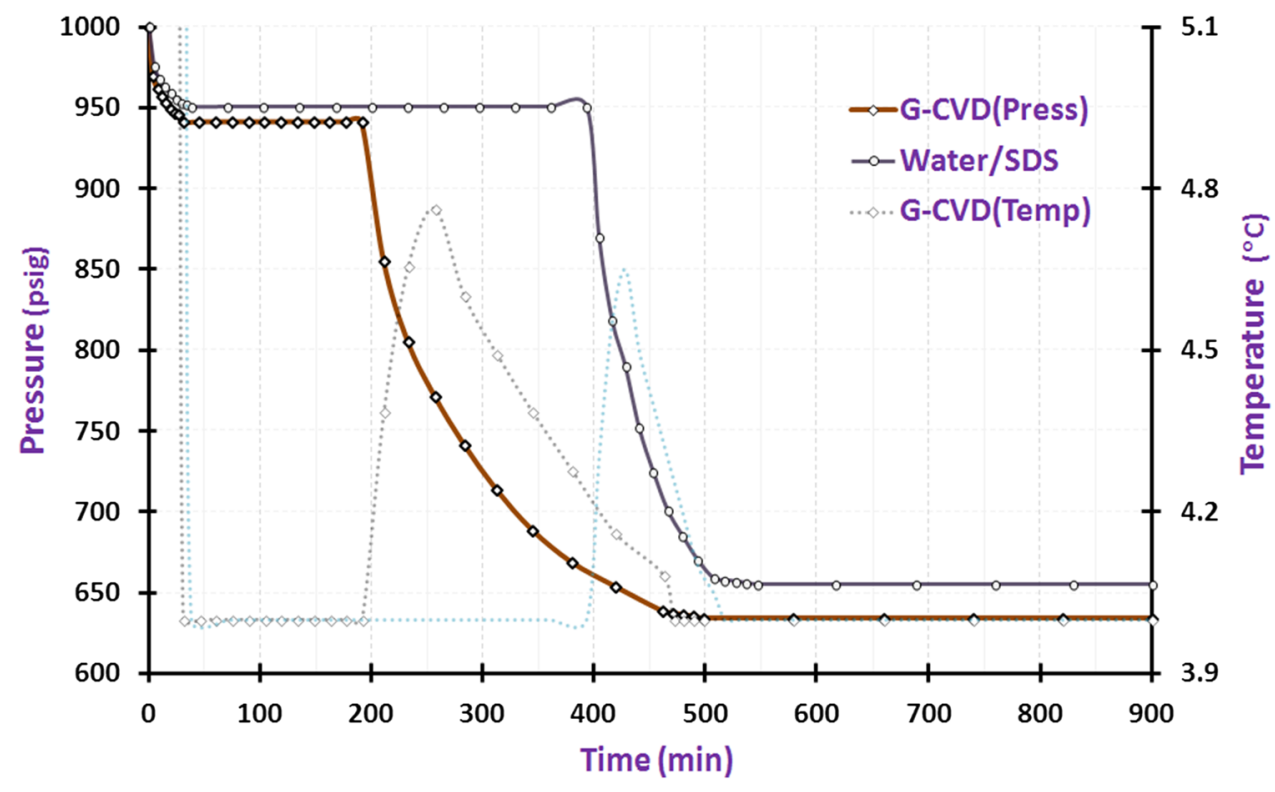


circulating coolant. Calculations showed that the induction time in presence of graphene nano-sheets is reduced $54.7 \%$ compared to the blank sample. This reduction can be caused by presence of nanometric heterogeneous nucleons, preparing more active sites for nucleation phenomenon. Besides, graphene nano-fluid, as fluid consists of two-dimensional angular nano-particles, enhance the heat transfer coefficient of the fluid; making the heat generated in reactor exit more efficiently. The second pressure drop in the reactor took $271 \mathrm{~min}$ which was caused by hydrate formation. After that, pressure of the system was relatively constant at 639 psig over the time, being reduced 4 psig every $35 \mathrm{~min}$ that is negligible. Therefore, HFP duration is $462 \mathrm{~min}$ and equilibrium pressure is $639 \mathrm{psig}$. The amount of gas remained in the reactor in this pressure was calculated $0.98 \mathrm{~mol}$.

Storage capacity and hydrate stability

Using the composition of the natural gas used in HFP, molecular weight and density of the hydrates were calculated $17.8 \mathrm{~kg} / \mathrm{kmol}$ and $910.41 \mathrm{~kg} / \mathrm{m}^{3}$, respectively. By

Table 1 Comparison of storage capacity of graphene nano-sheets/ water with SDS/water

\begin{tabular}{lll}
\hline Sample & SDS/water & Graphene/water \\
\hline Equilibrium pressure (psig) & 655.1 & 634.5 \\
Mole of gas remained & 1.016 & 0.980 \\
Mole of gas hydrated & 0.571 & 0.607 \\
Mole fraction of gas in hydrate & 0.103 & 0.110 \\
Storage capacity & 124.3 & 133.7 \\
Increased storage $(\%)$ & - & 7.6 \\
\hline
\end{tabular}

substituting these data in Eq. (5), storage capacity will be calculated using Eq. (6) as follows:

$V / V_{\mathrm{H}}=1209 c$

Storage capacity of the hydrates formed in presence of graphene nano-sheets has been calculated and compared with the blank sample in Table 1.

It is observed that storage capacity is increased $7.6 \%$ in presence of graphene nano-sheets, enhancing it from 124.3 up to 133.7. This enhancement may be due to the increased dissolution of gas in water. Graphene nano-sheets have also increased heterogeneous active sites, causing nucleation process to proceed faster so that more amount of gas can be stored within water molecules cavities.

To study the stability of the hydrates, temperature of the system was decreased to $-10^{\circ} \mathrm{C}$ after the process finished and remained in that temperature for $10 \mathrm{~h}$. The relief valve was then opened until pressure of the system reached 1 atm; causing non-hydrated gas exits the system. The relief valve was closed afterwards and pressure was measured instantaneously for $12 \mathrm{~h}$. By subtracting the number of moles released using Eq. (1) from the total number of moles stored in hydrates, the percentage of hydrate decomposition was calculated. Figure 7 shows the comparison between percentage of hydrate decomposition in SDS/ water and graphene/water.

As it is observed in Fig. 7 after $10 \mathrm{~h}$, maximum hydrate decomposition in presence of graphene nano-sheets was $11.9 \%$. It is also observed that the decomposition had occurred just in the first $7 \mathrm{~h}$, however, this process took $9 \mathrm{~h}$ in blank sample. Hydrate decomposition percentage of graphene/water and SDS/water over the time is compared in Table 2.
Fig. 7 Comparison between percentage of hydrate decomposition in SDS/water and graphene/water

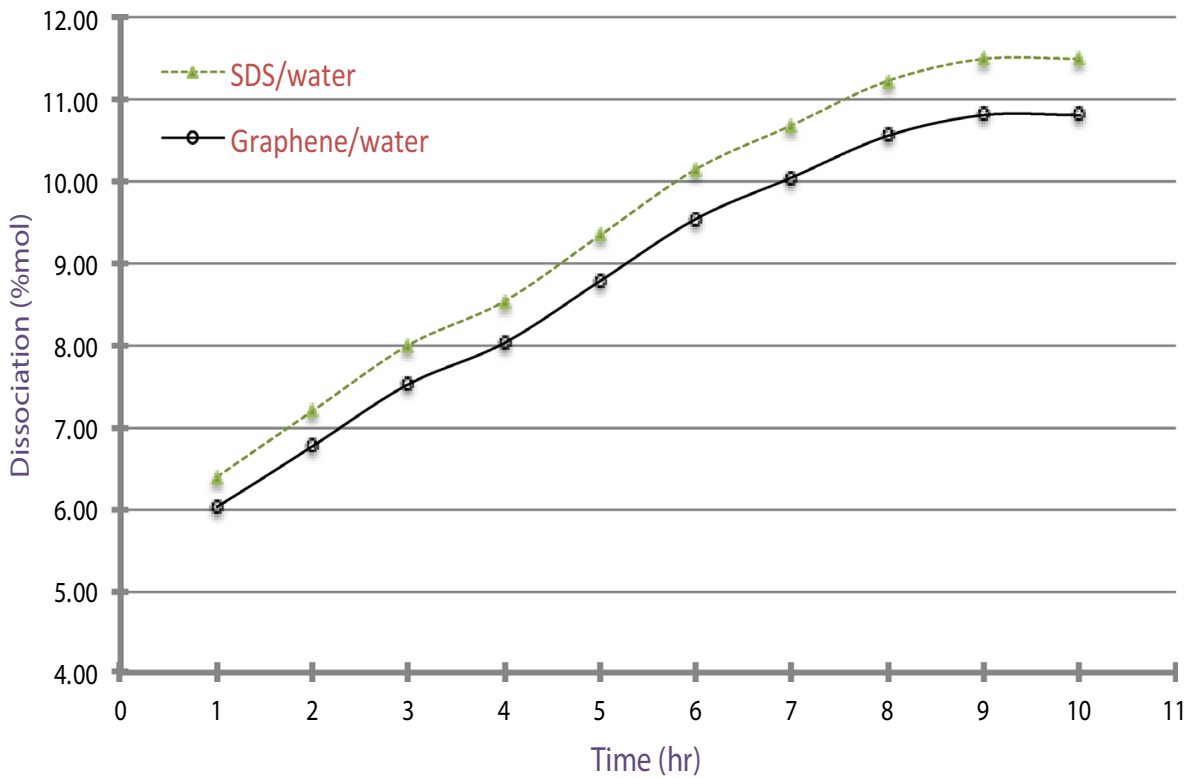


Table 2 Comparison of hydrate decomposition percentage over the time; graphene/water and SDS/water

\begin{tabular}{lllllllllll}
\hline Time (h) & 1 & 2 & 3 & 4 & 5 & 6 & 7 & 8 & 9 & 10 \\
\hline Blank sample & 6.39 & 7.12 & 8.00 & 8.53 & 9.34 & 10.14 & 10.68 & 11.22 & 11.49 & 11.49 \\
Graphene nano-fluid & 6.01 & 6.76 & 7.52 & 8.02 & 8.78 & 9.54 & 10.04 & 10.55 & 10.80 & 10.80 \\
\hline
\end{tabular}

It is observed in Table 2 that hydrate decomposition has been continued after $7 \mathrm{~h}$ in SDS/water sample; however, it is stopped at $7 \mathrm{~h}$ in presence of graphene nano-sheets. Therefore, it is concluded that the stability of hydrates is increased in presence of graphene. This fact may be due to the strength of hydrate network created by adding graphene to the water; causing its resistance against decomposition.

It should be also considered that use of SDS had definitely affected the response by reducing the interphase mass transfer resistance at the gas/liquid interface and increasing the methane mole fraction in the bulk liquid during hydrate growth. But using graphene beyond the SDS, shows to increase storage capacity and stability of the hydrates while decreasing the induction time. This facilitation is likely caused by two reasons: (1) using graphene creates a nano-fluid with higher heat transfer coefficient [21] which enhances the heat transfer to the jacket of the reactor during the hydrate growth. (2) Surface to volume ratio increases in nanometric dimensions, preferring a larger active sites available for nucleation process.

\section{Conclusion}

Storage capacity is increased $6.7 \%$ in presence of graphene nano-sheets. It is observed that not only the decomposition of hydrates increased but also it was decreased although the amount of water available for participating in self-protection process in presence of graphene/water was less than that for SDS/water sample. Therefore, graphene nano-sheets increased stability of the hydrates while increasing the storage capacity.

It is also observed that graphene nano-sheets play the same role of surfactant in gas storage process with the difference of increasing the stability; however, surfactants reduce stability and increase the decomposition. Therefore, graphene nano-sheets can be used as an alternative of the surfactants, because they have improved the HFP in any stage while increasing hydrate stability.

Acknowledgments This research was partly financed by the Iran National Science Foundation (INSF), to which the authors are very grateful. In addition, TPNT cooperation is highly appreciated.

Open Access This article is distributed under the terms of the Creative Commons Attribution License which permits any use, distribution, and reproduction in any medium, provided the original author(s) and the source are credited.

\section{References}

1. Thomas, S., Dawe, R.A.: Review of ways to transport natural gas energy from countries which do not need the gas for domestic use. Energy 28, 1461-1477 (2003)

2. Gudmundsson, J.S., Hveding, F., Borrehaug, A.: Transport of natural gas as frozen hydrate, 5th international offshore and polar engineering conference, Hague (1995)

3. Gudmundsson, J.S., Brrehaug, A.: Frozen hydrate for transport of natural gas. 2nd international conference on natural gas hydrate, Toulouse (1996)

4. Miata, K., Okui, T., Hirayama, H., Ihara, M.: A challenge to highrate industrial production of methane hydrate. 4th international conference on gas hydrates, Yokohama (2002)

5. Takaoki, T., Iwasaki, T., Katoh, Y., Arai, T., Horiguchi, L.: Use of hydrate pellets for transformation of natural gas-I: advantage of pellet form of natural gas hydrate in sea transportation. 4th international conference on gas hydrates, Yokohama (2002)

6. Verrett, J., Posteraro, D., Servio, Ph: Surfactant effects on methane solubility and mole fraction during hydrate growth. Chem. Eng. Sci. 84, 80-84 (2012)

7. Mohammadi, A., Manteghian, M., Haghtalab, A., Mohammadi, A.H., Rahmati Abkenar, M.: Kinetic study of carbon dioxide hydrate formation in presence of silver nanoparticles and SDS. Chem. Eng. J. 237, 387-395 (2014)

8. Zhong, Y., Rogers, R.: Surfactant effects on gas hydrate formation. Chem. Eng. Sci. 55, 4177-4187 (2000)

9. Sun, Z., Wang, R., Ma, R., Guo, K., Fan, S.: Natural gas storage in hydrate with the presence of promoters. Energy Convers. Manag. 44, 2733-2742 (2003)

10. Guo, Y., Fan, S., Guo, K., Chen, Y.: Storage capacity of methane in hydrate using calcium hypochlorite as additive. 4th international conference on gas hydrates, Yokohama (2002)

11. Sun, Z., Ma, R., Wang, R., Guo, K., Fan, S.: Experimental studying of additives effects on gas storage in hydrate. Energy Flues 17, 1180-1185 (2003)

12. Ganji, H., Manteghian, M., Rahimi mofrad, H.: Effect of mixed compounds on methane hydrate formation and dissociation rates and storage capacity. Fuel Process. Technol. 88, 891-895 (2007)

13. Lin, W., Chen, G.J., Sun, C.Y., Guo, X.Q., Wu, Z.K., Liang, M.Y.: Effect of surfactants on the formation and dissociation behavior of methane hydrate. Chem. Eng. Sci. 59, 4449-4455 (2004)

14. Ganji, H., Manteghian, M., Sadaghiani, K., Omidkhah, M.R., Rahimi mofrad, H.: Effect of different surfactants on methane hydrate formation rate, stability and storage capacity. Fuel $\mathbf{8 6}$, 434-441 (2007)

15. Ganandran, N., Amin, R.: The effect of hydrotropes on gas hydrates formation. J. Pet. Sci. Eng. 40, 37-46 (2003)

16. Han, X., Wang, S., Chen, X., Liu, F.: Surfactant accelerates gas hydrate formation. 4th international conference on gas hydrates, Yokohama (2002)

17. Karaaslan, U., Uluneye, E., Parlaktuna, M.: Effect of an anionic surfactant on different type of hydrate structures. J. Pet. Sci. Eng. 35, 49-57 (2002)

18. Yoshikawa, K., Kondo, Y., Kimura, T., Fujimoto, T.: Production method for hydrate and device for proceeding the same 1. U.S. patent, 12-23 (2003) 
19. Manteghian, M., Mousavi Safavi, S.M., Mohammadi, A.: The equilibrium conditions, hydrate formation and dissociation rate and storage capacity of ethylene hydrate in presence of 1,4dioxane. Chem. Eng. J. 217, 379-384 (2013)

20. Park, S., An, E., Lee, S., Chun, W., Kim, N.: Characteristics of methane hydrate formation in carbon nanofluids. J. Ind. Eng. Chem. 18, 443-448 (2013)

21. Ghozatloo, A., Shariaty-Niasar, M., Rashidi, A.M.: Preparation of nanofluids from functionalized graphene by new alkaline method and study on the thermal conductivity and stability. Int. Commun. Heat Mass Transfer 42, 89-94 (2013)

22. Watanabe, K., Niwa, S., Mori, Y.H.: Surface tensions of aqueous solutions of sodium alkyl sulfates in contact with methane under hydrate-forming conditions. J. Chem. Eng. Data 50, 1672-1676 (2005)

23. Lo, C., Zhang, J.S., Couzis, A., Somasundaran, P., Lee, W.: Adsorption of cationic and anionic surfactants on cyclopentane hydrates. J. Phys. Chem. C 114, 13385-13389 (2010)

24. Lo, Ch., Zhang, J., Somasundaran, P., Lee, J.W.: Investigations of surfactant effects on gas hydrate formation via infrared spectroscopy. J. Colloid Interface Sci. 376, 173-176 (2012)

25. Banks, C.E., Compton, R.G.: New electrodes for old: from carbon nanotubes to edge plane pyrolytic graphite. Analyst 131, 15-21 (2006)

26. Torabi Kachoosangi, R., Banks, C.E., Compton, R.G.: Graphite impurities cause the observed 'electrocatalysis' seen at C60 modified glassy carbon electrodes in respect of the oxidation of 1-cysteine. Anal. Chim. Acta. 566, 1-4 (2006)

27. Sljukić, B., Banks, C.E., Compton, R.G.: Iron oxide particles are the active sites for hydrogen peroxide sensing at multiwalled carbon nanotube modified electrodes. Nano Lett. 6, 1556-1558 (2006)

28. Guoxiu, W., Juan, Y., Park, J., Xinglong, G., Wang, B., Liu, H., Yao, J.: Facile synthesis and characterization of graphene nano sheets. J. Phys. Chem. C 112, 8192-8195 (2008)

29. Fu, M., Jiao, Q., Zhao, Y., Lia, H.: Vapor diffusion synthesis of $\mathrm{CoFe}_{2} \mathrm{O}_{4}$ hollow sphere/graphene composites as absorbing materials. J. Mater. Chem. A 2, 735-744 (2014)
30. Geng, Y., Wang, S.J., Kim, J.K.: Preparation of graphite nanoplatelets and graphene sheets. J. Colloid Interface Sci. 336, 592-598 (2009)

31. Chen, J., Li, Y., Huang, L., Li, Ch., Shi, G.: High-yield preparation of graphene oxide from small graphite flakes via an improved Hummers method with a simple purification process. Carbon 81, 826-834 (2015)

32. Bule, Sh.S, Kumbhare, M.R., Dighe, P.R.: Synthesis and in vitro biological evaluation of a novel series of 4-(Substituted)-5methyl-2-phenyl-1, 2-dihydro-3H-pyrazol-3-one as antioxidant. J. Chem. Biol. Phy. Sci. 3, 1996-2005 (2013)

33. Lia, M., Guoa, W., Lib, H., Xuc, Sh, Qua, C., Yanga, B.: Synthesis of chemical vapor deposition graphene on tantalum wire for supercapacitor applications. Appl. Surf. Sci. 317, 1100-1106 (2014)

34. Wojtoniszak, M., Mijowska, E.: Controlled oxidation of graphite to graphene oxide with novel oxidants in a bulk scale. J. Nanopart. Res. 14, 1248 (2012)

35. Hwang, Y., Lee, J.K., Lee, C.H., Jung, Y.M., Cheong, S.I., Lee, C.G., Ku, B.C., Jang, S.P.: Stability and thermal conductivity characteristics of nanofluids. Thermochim. Acta. 455, 70-74 (2007)

36. Jin, H., Xianju, W., Qiong, L., Xueyi, W., Yunjin, Z., Liming, L.: Influence of $\mathrm{pH}$ on the stability characteristics of nanofluids in: symposium on photonics and optoelectronics. SOPO, 1-4 (2009)

37. Carroll, J.: Natural gas hydrates: a guide for engineers, 2nd edn, Gulf Professional, USA, (2009), ISBN-10: 0750684909

38. Sloan, E.D., Carolyn Koh, J.: Clathrate Hydrates of Natural Gases, 3rd edn. CRC Press, Boca Raton (2008)

39. Okutani, K., Kuwabara, Y., Mori, Y.H.: Surfactant effects on hydrate formation in an unstirred gas/liquid system: an experimental study using methane and sodium alkyl sulfates. Chem. Eng. Sci. 63, 183-194 (2008) 